\title{
Author Correction: Non-collinear spin states in bottom-up fabricated atomic chains
}

\author{
Manuel Steinbrecher ${ }^{1,2}$, Roman Rausch ${ }^{3}$, Khai Ton That ${ }^{1}$, Jan Hermenau', Alexander A. Khajetoorians ${ }^{1,2}$ \\ Michael Potthoff ${ }^{3}$, Roland Wiesendanger ${ }^{1} \&$ Jens Wiebe (i) ${ }^{1}$
}

Correction to: Nature Communications https://doi.org/10.1038/s41467-018-05364-5; published online 20 July 2018

The original version of this Article contained an error in Fig. 3 in which Fig. 3b and Fig. 3e incorrectly duplicated Fig. 2a and Fig. 2d, respectively. This has now been corrected in both the PDF and HTML versions of the Article.

Published online: 19 November 2018

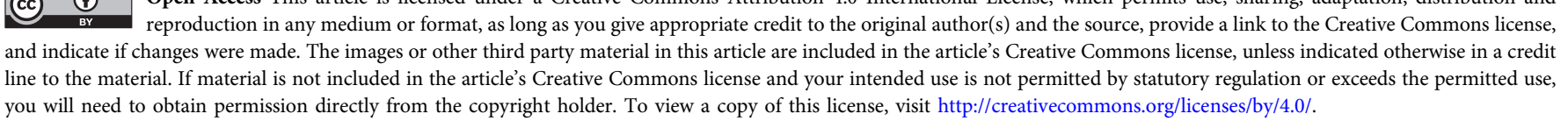

(C) The Author(s) 2018

\footnotetext{
${ }^{1}$ Department of Physics, Hamburg University, Jungiusstrasse 9A, 20355 Hamburg, Germany. ${ }^{2}$ Institute for Molecules and Materials (IMM), Radboud University, P.O. Box 9010//0786500 GL Nijmegen, The Netherlands. ${ }^{3}$ I. Institute for Theoretical Physics, Hamburg University, Jungiusstrasse 9, 20355 Hamburg, Germany. Correspondence and requests for materials should be addressed to M.S. (email: manuel.steinbrecher@physik.uni-hamburg.de) or to R.R. (email: rrausch@physnet.uni-hamburg.de) or to J.W. (email: jwiebe@physnet.uni-hamburg.de)
} 